ISSN 1518-3483

\title{
La educación virtual como la modalidad educativa para las personas con necesidades especiales: solo en la red no hay personas con discapacidad
}

\section{Virtual education as a means of education for people with} special needs: only on social network there are no disabled people

\section{Claudio Rama}

\section{Resumén}

La problemática del acceso a la educación para las personas con discapacidad, tradicionalmente ha sido vista desde el enfoque de la "Educación Especial", el cual nunca se incorporó a la Educación Superior, la cual que siempre atendió igual a todos los estudiantes. Fue recién en los años 90 cuando tal paradigma cambió para centrarse en la accesibilidad, a través de políticas y estrategias centradas en superar las barreras 
físicas y educativas, e impulsar políticas de compensación. Tales políticas aunque permitieron aumentar el acceso para algunas personas, no cubrieron el acceso de todos, y se expresaron en altas tasas de deserción y baja eficiencia de titulación para las personas con discapacidades. El presente ensayo analiza esa fase y los sus resultados en bajos niveles de accesibilidad en la región, y se centra en cómo la actual virtualización está cambiando dicho paradigma al superar la problemática de la accesibilidad y centrarse en el acceso digital. En tal sentido, se sostiene que el aprendizaje colaborativo en red y el hogar como aula digital, se constituyen en la única modalidad que efectivamente permite la total accesibilidad para todas las personas con discapacidad, más allá de la diversidad de situaciones y patologías, y analizar como bajo el mundo digital no hay personas con discapacidades en tanto los soft y los hardware se constituyen en los interfaces de la accesibilidad a la educación.

Palabras-clave: Personas con discapacidad. Acceso. Educación Superior. Virtualización. Educación a Distancia.

\section{Abstract}

The problem of access to education for people with disabilities has traditionally been viewed from the standpoint of "Special Education", which was never incorporated into Higher Education, which always attended equally to all students. It was only in the 90s when that paradigm shifted to focus on accessibility, through policies and strategies focused on overcoming the physical and educational barriers, and boost compensation policies. Such policies increase access but allowed for some people, not covered access for all, and is expressed in high dropout rates and low efficiency of qualification for persons with disabilities. This paper analyzes the phase and results in low levels of accessibility in the region, and focuses on how virtualization is changing the current paradigm to overcome the problems of accessibility and focus on digital access. In this regard, it is argued that collaborative learning and home network as digital classroom constitute the only mode that actually allows full accessibility for all people with disabilities, beyond the diversity of situations and conditions, and analyze as under the 
digital world there are no people with disabilities in both the software and hardware interfaces constitute the accessibility to education.

Keywords: People with disabilities. Access. Higher Education. Virtualization. Distance Education.

\section{La exclusión en la Educación Superior}

La problemática de la exclusión en Educación Superior en lo atinente a su acceso, permanencia y egreso era analizada tradicionalmente como consecuencia de las desigualdades económicas y sociales externas a las instituciones universitarias. Es claro que los factores externos eran determinantes de la desigualdad: los escasos presupuestos dedicados a la educación superior; la baja calidad de la Educación Media pública, también asociada a bajos presupuestos; la injusta distribución nacional del ingreso; los costos y la mercantilización de la Educación Superior, la incapacidad de vastos sectores de pagar las matrículas, los costos indirectos de los estudios, la heterogeneidad socioeconómica o la localización especial. Los ingresos de los hogares y el stock cultural era los determinantes para explicar los recorridos educativos y las oportunidades escolares, en tanto factores de mayor peso a la hora de explicar las desigualdades educativas, las cuales se ensanchan a medida que se avanza en los ciclos educativos sucesivos. Sin embargo, los relevamientos comenzaron a detectar que la deserción y abandono tenían también múltiples determinantes intrínsecos a la vida y desenvolvimiento de las instituciones que coadyuvaban a reproducir las desigualdades y exclusiones existentes en las sociedades (CINDA, 2005).

Ello contribuyó a que desde los 90 hubiese un giro en el foco de atención, y los análisis se comenzaron a orientar hacia su focalización al interior de las propias instituciones de Educación Superior con el objetivo de profundizar en como su dinámica de funcionamiento reproducía los niveles de exclusión de sus respectivas sociedades. La preocupación comenzó a centrarse en distinguir cuáles podían ser las políticas que podían 
permitir para que la Educación Superior alcance a todos. En la accesibilidad física y educativa recayó tal enfoque, que mostró la importancia de las limitaciones propias de las instituciones para la inclusión, destacándose las estructuras de gestión poco flexibles, sistemas pedagógicos tradicionales, mecanismos selectivos de ingreso, ausencia de políticas que contrarresten los procesos de inequidad previos o la carencia de un seguimiento y atención particulares a los estudiantes con dificultades.

También se destaca la gran autonomía de algunas instituciones que les permiten seleccionar a sus alumnos y profesores, con lo cual provocan que los estudiantes más rezagados sean expulsados del sistema o que se inclinen hacia instituciones terciarias que brindan menores niveles de calidad, en una dinámica que reproduce finalmente las desigualdades existentes.

La ausencia de políticas públicas compensatorias y la rápida expansión de nuevos saberes y conocimientos a escala global, que tornan más exigentes y selectivos los circuitos académicos, contribuían también a restringir el acceso y a dificultar la permanencia a vastos sectores que intentan formarse en el nivel superior, y que presentan rezagos culturales, limitaciones de accesibilidad y por ende requerimientos de compensaciones educativas y económicas.

\section{El acceso como paradigma de la inclusión y las diversas fases}

Desde los '90, la preocupación por el acceso de las personas con discapacidades a la Educación Superior se comenzó a constituirse en un tema central en la agenda de la Educación Superior y se focalizó en los temas normativos para permitir la accesibilidad física en tanto se concebía que dichas barreras eran las centrales para responder a sus necesidades especiales. La accesibilidad física fue la primera fase en la cual se expresó la lógica de la inclusión.

En 1993, las Naciones Unidas publicaron las "Normas Uniformes sobre Equiparación de Oportunidades para las Personas con Discapacidad" que plantearon estrategias para modificar el entorno como bases de 
las acciones para la equiparación de oportunidades junto a acciones para la eliminación de la discriminación. Su aprobación "rompió con el paradigma asistencial al romper con la idea que el problema es la persona para centrar el problema en el entorno (JIMÉNEZ SANDOVAL, 2002). Este nuevo abordaje implico un giro para la educación de esta población e derivó en la promulgación de leyes de igualdad de oportunidades para las personas con discapacidad en la región que imponían obligaciones para las entidades públicas y privadas. La accesibilidad física, sin embargo pronto verificó la existencia al interior del mundo educativo y de las propias instituciones, de una cultura discapacitante que mantiene una marcada distancia entre personas según sus condiciones o disfunciones físicas y en la cual tiene un rol destacado la ausencia de pedagogías, equipos y capacitación del personal para cubrir esas necesidades y atender con éxito a este sector de la población (VENEZUELA, 2004). Ello condujo a una segunda fase en la lógica de la inclusión dada por la accesibilidad a los entornos educativos, y que focalizo la superación en las adecuaciones curriculares, la incorporación de la temática de la discapacidad en los programas de estudio, la formación de profesionales especializados en el tema, y la existencia de recurso didácticos focalizados en las necesidades especiales (como bibliotecas braille por ejemplo) etc.

Las nuevas exigencias pusieron a los centros de Educación Superior bajo el reto de facilitar la accesibilidad para las personas con discapacidad y ello a su vez abrió contribuyó a encarar la problemática de cómo construir procesos de enseñanza, recursos instruccionales y aprendizajes en la diversidad, lo cual coadyuvó a plantear el cambio de una problemática centrada en la educación a una focalizada en el aprendizaje.

La Conferencia Mundial sobre la Educación Superior (CMES), convocada por la Unesco en 1998, planteó que el acceso al desarrollo educativo debía estar basado en los méritos, la capacidad y los esfuerzos personales, y que no se podía admitir ningún tipo de discriminación vinculada a la raza, el sexo, el idioma o la religión, ni tomar en consideración aspectos económicos, culturales, sociales, ni tampoco las diversas capacidades físicas. La CMES sugirió que la equidad en el acceso debía empezar por el fortalecimiento de la educación media en el marco de un sistema continuo y, entendiendo que 
no hay país donde no se establezcan requisitos para el ingreso, sostuvo que en toda política de acceso se debe dar preferencia a los méritos y superar las barreras. Sin embargo, dada la heterogeneidad de nuestras sociedades y la existencia de circuitos de escolarización diferenciales, se consideró entonces, que además de accesibilidad física y accesibilidad educativa, se requería también una discriminación proactiva hacia las personas con discapacidad atenta al objetivo de equidad de la política pública. Esta se conformó como la tercera fase de la accesibilidad para las personas con discapacidad. Tal esquema se basó sin embargo en la accesibilidad al modelo presencial que se asumió como el objetivo de la inclusión.

\section{El fracaso de un tipo de enfoque sobre la inclusión}

Los datos indican que más allá de la profundidad y amplitud de las fases anteriores aún en curso, la inclusión para las personas con discapacidad es altamente limitada. La matrícula de la Educación Superior, durante los últimos 10 años en América Latina y el Caribe se duplicó y alcanzó una cobertura del $41 \%$ con más de 24 millones de estudiantes. Sin embargo tal violenta expansión de la matrícula no significó que se hayan resuelto las exclusiones a las personas con necesidades especiales sino que los datos verifican la permanencia de las desigualdades de acceso. Muchas leyes de accesibilidad se han aprobado en estos años y se han ido recorriendo muy lentamente las tres fases referidas, con logros relativamente escasos.

Un estudio por ejemplo sobre la Educación Superior y las personas con discapacidades en Colombia del Departamento Administrativo Nacional de Estadística (DANE) evidenció que el 6.3\% de la población colombiana presenta limitaciones permanentes, y que de ese porcentaje, el 33.3\%; no tiene nivel educativo, el 29.1\% tiene nivel de básica primaria incompleta, que el $2.34 \%$ tiene algún nivel de Educación Superior, ya sea técnica, tecnológica o profesional; que el $1 \%$ de las personas tiene culminados sus estudios superiores y el $0.1 \%$ ha cursado postgrados, mostrando la alta desigualdad de este grupo social respecto a la distribución 
normal de la población total en los distintos niveles educativos al tiempo que un aumento de la exclusión a medida que aumentan los estudios derivados de la deserción y el abandono (MATERON PALACIOS; MOLINA BEJAR, PARRA DUSSAN, 2010) ${ }^{1}$. En Brasil los estudios muestran que para el 2005 apenas el 0,27\% de los estudiantes con necesidades especiales están insertos en el nivel superior a pesar de que el 14,4\% de la población en el Censo Demográfico del 2000 manifestaba alguna dificultad de escuchar, ver, trasladarse o mental (REZENDE, 2009). En México sólo 4 de cada 100 personas con discapacidad, el 0,4\%, acceden a la Educación Superior (CAMACHO REAL; VARELA NAVARRO, 2006). Más allá de la debilidad de la información al interior de las universidades, es de destacar que tal problemática además tienden a aumentar dado el incremento a escala global del número de personas con discapacidad debido al envejecimiento de la población que trae un mayor riesgo de discapacidad por el incremento global de los problemas crónicos de salud asociados a la discapacidad, como la diabetes, las enfermedades cardiovasculares y los trastornos mentales (ORGANIZACIÓN MUNDIAL DE LA SALUD - OMS; BANCO MUNDIAL, 2011). El aumento de la edad media de los estudiantes de Educación Superior, la expansión de los niveles y la irrupción de la educación a lo largo de la vida, incentiva también la problemática de la accesibilidad a la Educación Superior.

El ingreso a la Educación Superior excede las cuestiones meramente técnicas, y engloba una amplia cantidad de elementos y de variables sociales, políticas, económicas y académicas. La restricción a la cobertura para estos sectores inclusive se da tanto frente a mecanismos de selección a través de los exámenes como con sistemas de accesos sin restricciones implícitas, lo cual plantea considerar que las limitaciones no vienen sólo de las instituciones, de sus infraestructuras edilicias y académicas, sino sin duda de la propia modalidad presencial.

1 A partir de datos del Departamento Administrativo Nacional de Estadística-DANE-, Ministerio de Educación Nacional-MEN-. Registro para la localización y caracterización de la población con discapacidad. In. Bogotá DC; 2002 
Es claro que la selección a través de pruebas, de rendimientos históricos (o combinaciones de ambos) desfavorece a los estudiantes que vienen de circuitos escolares de bajo rendimiento. Esto es mucho más significativo en sociedades donde el sector privado en el nivel medio ofrece una mayor calidad educativa que el sector público, así como una mejor capacidad de retención, pero los problemas de acceso real han tendido a superar esas barreras mediante políticas preactivas, pero a su vez muchos de los logros del ingreso han derivado no han tenido los mismos éxitos en lo atinente al egreso, sino que se han producido altos niveles de deserción y abandono en los sistemas abiertos para esta población. Sin duda que el principal obstáculo para que las personas con discapacidades no accedan a la Educación Superior es que en su mayoría tampoco concluyen la etapa correspondiente a la formación secundaria, por lo que inclusive la resolución exitosa de esta problemática no se puede reducir simplemente al acceso al nivel terciario, sino que también es indispensable considerar las políticas académicas y de asistencia previas.

En los últimos años los bajos niveles de cobertura de las personas con discapacidad se han mantenido y se ha constatado la persistencia de las exclusiones, aún a pesar de los marcos normativos, los cambios físicos o los accesos proactivos. Los fracasos más allá de la incidencia de los recursos permiten identificar que el problema no se reduce ellos, sino fundamentalmente a la falta de dinámicas educativas atentas a las diversidades y la ausencia de tecnologías que permitan esas atenciones especiales, y en fundamentalmente en las limitaciones de la propia tecnología de la educación presencial. Algunos definen esta dinámica de accesibilidad como una accesibilidad cosmética.

El entorno no es sólo el espacio físico de la institución, es también el aula y el modelo educativo presencial. Más allá de focalizar la atención en las características propias de las instituciones universitarias que muestran distintas resistencias culturales, económicas, administrativas y físicas a la incorporación de los diferentes sectores excluidos de la sociedad, la problemática se plantea también en relación a las limitaciones que genera el modelo educativo dominante de tiza, lengua, pizarrón, 
laboratorios, bibliotecas y campus universitarios. Este modelo atiende un tipo de población estudiantil y no se ajusta con igual eficacia a todos los estudiantes por sus propias necesidades especiales. Inclusive aún a pesar de un relativo mejoramiento en la accesibilidad, no alcanza la total inclusión por las carencias de una atención más flexible y particularizada en el modelo presencial, más allá de la ausencia de tecnologías específicas para atender problemáticas del aprendizaje de las personas con discapacidad o inclusive de otros grupos como las personas privadas de la libertad. Por ello se constata que la dinámica universitaria continua reproduciendo las exclusiones a pesar de poder resolverse en algunos casos la mera accesibilidad al aula. Se requiere entonces la formulación de políticas que permitan la inclusión de todos en función de los méritos que tengan los aspirantes, y ajustadas a compensar sus carencias. Así, aún a pesar de la importancia de la construcción de políticas asistencialistas públicas ${ }^{2}$, de dinámicas pedagógicas compensatorias o de marcos legales específicos que ayuden a cimentar dinámicas universitarias más inclusivas, hay limitaciones que provienen desde la educación presencial tradicional en tanto modelo homogéneo, centrado en aulas, y que impone un paradigma educativo no diferenciado ni individualizado y que tiende a ser catedrático, memorístico y repetitivo. El cambio sólo del ambiente físico y no del ambiente educativo no logra la accesibilidad para esta población.

La marginación excede el aspecto económico y edilicio pues existe un tipo de exclusión que afecta a una parte de los estudiantes con discapacidades físicas, quienes no logran ingresar a la Educación Superior a pesar de pertenecer, incluso, a los quintiles superiores de ingreso o tener altos stocks de capital cultural. Sin duda las instituciones de Educación Superior no cuentan aún con las prácticas pedagógicas, las políticas proactivas, los equipos técnicos, las infraestructuras completas adaptadas a las diversidades de situaciones de discapacidades, la capacitación del personal destinado a la atención individualizada de esta población así como una legislación que

2 Estas políticas a su vez se han basado en el desarrollo del concepto el derecho a la asistencia y del asistencialismo mínimo. 
favorezca una fácil accesibilidad. La solución de la accesibilidad cuando se da es inclusive para pocos y también tiene enormes exigencias personales que finalmente determinan altas tasas de deserción y abandono de aquellos que logran acceder pero cuyo sobreesfuerzo continuo en el acceso a una educación presencial, los termina expulsando.

Son múltiples los factores que soportan esas realidades como la ausencia de normas o su incumplimiento por falta de recursos, o la carencia de tecnologías que permitan cubrir sus necesidades especiales. Si bien un sector podría acceder a aprendizajes cambiando parte del entorno sobre los mismos modelos de enseñanza, se constata que una porción sustancial de las personas con discapacidades, requieren una atención particularizada para su aprendizaje, en tanto es imposible para ellos compartir el proceso de enseñanza en los mismos ambientes de aprendizaje presenciales por los particularismos de sus necesidades especiales. Históricamente el enfoque para superar la exclusión se dio a través de una educación especial segregada, y se basó en una visión que planteaba que para superar las situaciones de minusvalía se requerían acciones proactivas específicas que permitieran compensar las carencias existentes de la dinámica educativa masificada.

Durante los últimos años nuevos enfoques inclusivos han promovido una sustancial transformación del concepto tradicional de "personas incapacitadas" para avanzar hacia la definición de "personas con discapacidades" o "personas con capacidades especiales" con sus correspondientes necesidades para funcionar en el entorno social dominante. Estas concepciones han sido la base de centrar el tema en los entornos y fueron minando las bases de la "educación especial" que atendía diferenciadamente y en forma presencial a estos sectores y que en la educación básica o fundamental es aun dominante. Sin embargo, más allá de la problemática concreta de la salud, los nuevos paradigmas propendieron a reconocer las diversidades culturales, y apuestan a la valorización de esa diversidad por considerarla un elemento enriquecedor en el proceso de enseñanza y de aprendizaje.

La diferenciación institucional, curricular, pedagógica y estudiantil ha sido al tiempo el impulsor de la educación de la diversidad integrada en el aula, y que al tiempo permitió nuevas configuraciones a la educación y a la 
accesibilidad de estos sectores. Las teorías constructivistas e interactivistas, han mostrado el rol del aprendizaje como proceso colectivo de interacciones presenciales, y que ha sido el marco que ha valorado el rol de la diversidad y la integración social en los procesos de aprendizaje.

Desde el punto de vista social y hasta institucional, se reconoce que todas las personas tenemos características en común, pero a la vez presentamos particularismos y diversidades, que tornan a las diferencias entre los seres humanos como constantes y no excepcionales, pero desde el punto de vista educativo se reconoce el valor de la diversidad y la calidad en el aprendizaje (MEMBREÑO; HERRERA, 2000). Bajo este paradigma se dejó atrás la idea de una educación especial, ya que ella producía marginación y segregación de las personas con necesidades y capacidades especiales. Pero también se visualizaba que el abandono de esa fragmentación debía estar necesariamente acompañado por una transformación de las prácticas pedagógicas en las instituciones educativas en tanto ellas tenían modelos tradicionales que no dan cabida de manera conveniente a todas las diversidades.

Este complejo proceso, tuvo limitaciones en la Educación Superior donde se constató la ausencia de políticas públicas y universitarias que impulsaran medidas de compensación e integración que permitan alcanzar la igualdad de oportunidades para las personas con discapacidad. Así, la integración de los individuos con necesidades especiales en la Educación Superior muestra un sensible retraso, tanto en la formulación de marcos normativos como en su aplicación o en la formulación de prácticas sociales inclusivas ${ }^{3}$.

Aunque la realidad muestra que las universidades autónomamente no tienen comportamientos ni prácticas discriminatorios en relación con las personas con discapacidad, se verificaba que tampoco tienden

3 A un nivel universitario la Asociación Nacional de Universidades e Instituciones de Educación Superior (ANUIES) en México en coordinación con la Presidencia de la República ha formulado un marco de política universitaria en la materia. IESALC a comienzos de la década creó una Guía de evaluación de la accesibilidad física de las instituciones con el objeto que sirva como un instrumento para su acreditación. Sin embargo, la acción reguladora más importante ha sido la sanción de la Corte Suprema de Brasil que ha autorizado a las universidades a aplicar políticas proactivas de compensación en el acceso. 
a impulsar cambios significativos en sus dinámicas educativas que faciliten el acceso y la permanencia de estas personas. Inclusive en estos años aunque la accesibilidad física aumentó levemente, ello no se ha reflejado en incrementos significativos en el acceso y no son las personas con discapacidad que usas muchos de estas oportunidades.

Es necesario referir que la complejidad de la inclusión en el aula de diversidad de personas y de dinámicas de aprendizaje pertinentes para las distintas problemáticas personales, afecta los procesos de aprendizaje fuertemente homogenizados. Ello plantea la necesidad de visualizar tratamientos diferenciados a través de políticas específicas que permitan que quienes posean algún tipo de discapacidad no vean agravadas sus propias limitaciones debido a un entorno económico y social excluyente y que muchas veces por tratarlos igual, termina relegando a muchos de los participantes (CEDAC, 2004). Pero al tiempo necesita incluirlos y no marginarlos. Esta es sin duda una problemática difícil de resolver: la de integrar la diversidad y reconocer la especificidad, que entran en contradicción en el aula presencial y un modelo educativo homogéneo. La realidad ha sido de hecho la expulsión aún cuando se superaron las barreras de la accesibilidad física o de recursos de aprendizaje.

Es claro que las políticas aun no reduciéndose a los aspectos arquitectónicos o físicos que limitan el ingreso y permanencia de los asistentes, e incluyendo los aspectos pedagógicos y culturales, no logran sin embargo mitigar el abandono y la deserción en el contexto de la educación presencial. Ello más allá del limitado acceso a nuevas tecnologías de información, de la escasa formación en dinámicas interculturales de los docentes, la ausencia de normas específicas para la atención de las diversidades, de organismos de apoyo a los estudiantes con discapacidades o de insuficientes incentivos para el ingreso de docentes con discapacidades así como de la persistencia de prácticas sociales universitarias excluyentes (VEGA et al., 2005).

Las universidades han construido históricamente servicios sociales o sectores de apoyo para el cumplimiento de sus propias actividades. La creación de bibliotecas, laboratorios, comedores u hospitales universitarios, la inclusión de becas o de sistemas de transporte constituyen parte de una 
amplia gama de servicios y de políticas que se han ido desarrollando de manera autónoma para facilitar los accesos. Estas medidas permitieron cubrir demandas insatisfechas, y atender con mayor eficacia y favorecer la inclusión de diversos sectores excluidos. Esos servicios tradicionales de las universidades, han sido muy importantes y contribuyeron a producir cambios notables en el acceso, pero no han sido suficientes para abarcar a las personas con discapacidades y necesidades especiales por la alta diversidad de situaciones específicas. Es claro que los cupos o las rampas permiten un acceso menos dificultoso, pero las innovaciones arquitectónicas no bastan para contribuir a la permanencia, ya que no logran resolver la complejidad de los problemas educativos, requiriéndose modalidades pedagógicas inclusivas específicas que atienden los particularismos de todos los participantes, y entre ellas las de las personas con discapacidad ${ }^{4}$.

La existencia de un porcentaje de personas cuya deficiencias visuales, auditivas o motoras son de una reducida dimensión por lo cual relativamente pueden compartir los procesos de enseñanza en las aulas tradicionales en forma permanente. Una parte de estos no alcanza los niveles de aprendizaje necesarios. Otra parte logra acceder pero la complejidades que tienen son tales que finalmente abandonan y muestran también niveles menores aprendizaje. Pero hay inclusive una alta cantidad de ellos que requiere aulas y ambientes especiales de aprendizaje. Se calcula para el caso de Brasil que esta demanda de atención especial y diferenciada acontece en el $57 \%$ de los casos de ceguera, el $12 \%$ de los casos de baja visión, el 32\% de los casos de zurdez leve y el 56\% de los casos que fuera profunda, el 19\% en los casos de ceguera y zurdez; $2179 \%$ de los casos de deficiencia múltiple; el 31\% de los casos de deficiencia física y el $67 \%$ de los casos de autismo (BRASIL, 2006 apud REZENDE, 2009). Se excluyen los casos de deficiencia mental (68\%) o de Síndrome de Down (74\%) ya que ellos no alcanzan la Educación Superior, mientras que los otros casos,

4 Aún en relación a las rampas, el grado de inclinación definido siempre es una media y hay casos de personas con discapacidad que requerirían menos grados de inclinación. Toda solución presencial siempre es excluyente para alguna minusvalía específica. 
si pudieran seguir sus recorridos educativos si tuvieran los recursos y ambientes especiales de aprendizaje. La diversidad y los límites son medibles y en España, por ejemplo, los beneficios de la asistencia se asignan a aquellas personas a las cuales se les reconoce un grado de minusvalía igual o superior al 33 por ciento ${ }^{5}$. De hecho una evaluación igual puede medir en que intensidad una persona puede acceder a las instituciones presenciales y a sus métodos de enseñanza, o requerir nuevas modalidades de enseñanza particularizadas. Así, el acceso a la modalidad presencial tradicional, más allá de la superación de sus barreras, solo cubre una porción de las demandas de un sector de esta población que tiene altas diversidades de situaciones. Otra parte de sus miembros siempre requerirán una enseñanza especial y estará en riesgo de marginamiento.

La inclusión total requiere pasar de modalidades pedagógicas homogéneas, presenciales y catedráticas, a prácticas educativas de carácter interactivo, donde la posibilidad del aprendizaje sea flexible e individualizada y la educación se organice de manera que de respuesta a estas diversidades y donde el docente y las tecnologías cumplan el rol de ser tutor y recursos en un efectivo y posible autoaprendizaje con una enseñanza ajustada a sus necesidades y habilidades específicas.

Sin embargo, ello no es posible en tanto que las universidades carecen de la altísima diversidad de servicios de apoyo académico atentos a la enorme cantidad de discapacidades que pueden tener las personas, tales como lectores y grabadores de texto; adecuaciones tecnológicas para los exámenes; transcripciones en Braille; computadores con teclados y parlantes; equipamiento para ciegos, sordos y otras personas con minusvalías físicas, y de una lógica educativa despresencial. En ello también interactúa negativamente la falta de una formación pedagógica que les

5 El certificado de minusvalía (Discapnet) es un documento oficial que puede ser expedido únicamente por la Administración Pública. En este certificado ha de figurar si la minusvalía es temporal o definitiva, y quedará reflejado en ese mismo documento si la persona que ha solicitado dicho certificado ha de pasar revisión o no, y cuándo. Disponible en: <http://www. discapnet.es/Castellano/areastematicas/derechos/guiasrecursosderechos/Ciudadanos/Paginas/ Ciudadanos009\%20(2).aspx>. 
permita a los docentes trabajar con los estudiantes con discapacidades o sin ellas y establecer dinámicas educativas "a la carte", absolutamente individualizadas así como los niveles de formación de los docentes en Braille, lenguaje de señas para sordos, soroban etc.

Si bien las nuevas tecnologías han abaratado los costos de los equipos, la alta diversidad de situaciones no permite que las instituciones puedan adquirirlos para todas las potenciales personas. La ausencia de tales sistemas apoyo inclusive hace que algunas personas con discapacidades sólo puedan acceder a muy pocas opciones curriculares, a pesar de que con las oportunidades que brindan las tecnologías digitales cada vez más se puedan llevar adelante la inmensa mayoría de los estudios existentes.

\section{La virtualización como camino de accesibilidad e inclusión}

Un conjunto de causas explican el fracaso en la inclusión a las personas con discapacidad. Si bien faltan recursos y políticas, la estrategia referida de facilitar la inclusión a través de la accesibilidad física ha chocado contra las propias instituciones educativas que deben disponer ingentes presupuestos e inversiones muy superiores en relación al aporte de nuevos estudiantes, y una realidad dada por la ausencia de presión política por parte de los estudiantes con capacidades especiales, en tanto al estar fuera de dichas instituciones tienen escasa capacidad de presión sobre ella. La estrategia de encarar el problema de la inclusión en el entorno, para algunos la llamada inclusión cosmética de rampas, ha tenido lamentablemente escasa eficacia en resolver la inclusión. Inclusive las infraestructuras existentes tienen unos parámetros de accesibilidad bajos y por ende sólo es posible introducir cambios en el acceso con grandes reingenierías edilicias y educacionales. Pero aún a pesar de que existieran recursos la dinámica presencial no permite pedagogías diferenciadas de los procesos de autoaprendizaje ajustadas a sus necesidades específicas. La propia Organización Mundial de la Salud insiste en esta estrategia que visualizamos errónea y en su informe referido expresa que "eliminar los obstáculos en los espacios públicos, transporte, información y comunicación 
hará posible que las personas con discapacidad participen en la educación, empleo y vida social, reduciendo así su aislamiento y dependencia". Se centra casi exclusivamente en posibilitar el acceso a los sistemas y servicios convencionales, que sigue resultando una solución parcial, de muy largo plazo y que no valora las posibilidades de los ambientes digitales para los temas tanto educativos como laborares que crecientemente se expanden a través del teletrabajo (RIFKIN, 1996).

Las TIC permiten un nuevo enfoque educativo que centra parte de las soluciones a la inclusión educativa en la programación informática, los ambientes virtuales de aprendizaje, las redes virtuales de aprendizaje y los recursos digitales específicos desarrollados atendiendo a los particularismos presentes. Esta estrategia para facilitar el acceso, la permanencia y el egreso educativo está asociada a los ambientes recursos tecnológicos digitales a través de software específicos y al interior de redes y plataformas de aprendizaje virtuales que permitan responder con flexibilidad a los particularismos de las personas y al tiempo mantener los niveles de integración social. La sola incorporación de esos recursos al interior de las instituciones no resuelve el problema del acceso físico en las instituciones y la movilidad hacia ellas, ni los problemas de la compartir demandas diferenciadas de pedagogías distintas en la propia aula. La inclusión de las personas con discapacidad a través de las TIC, bajo el ambiente presencial no resuelve el problema de la accesibilidad física. Además tiene limitaciones asociadas a los problemas de traslado desde los hogares a las instituciones dado los entornos urbanos limitantes, a las complejidades de procesos de enseñanza en campos cada vez más especializados y que implican costos de equipamientos significativos y limitantes a la individualización del aprendizaje. Ellas abren el camino a la solución a través de la virtualización. El modelo de blended learning inclusive puede permitir también los espacios de convivencia que se pudieran requerir pero como complementos voluntarios del proceso de enseñanza y sujetos a las propias posibilidades de los participantes. La convivencia es en las redes sociales y de aprendizaje.

A través de aplicaciones informáticas, como por ejemplo el traductor automático de voz a texto del soft Dragon, o el traductor visual de voz a 
lenguaje de señas, el uso del laser para activar objeto, o el Mouse cibernético para el uso de cuatriplégicos por ejemplo, hacen que al interior de la red el ciego no es ciego, el sordo no es sordo y los que no se pueden trasladar se trasladan. En el espacio virtual no existe la discapacidad, y todas las personas están equiparadas en sus oportunidades ${ }^{6}$. Es este un camino que va construyendo políticas y estándares, las aplicaciones informáticas para accesibilidad de personas con discapacidad tienen ya un estándar dado por la Norma ISO 9241 que es aquella enfocada en la calidad, la usabilidad y la ergonomía tanto del hardware como del software. La norma evalúa en las páginas web múltiples elementos de accesibilidad a personas con discapacidades como por ejemplo la vista con contraste, la existencia de sintetizador de voz, el pasaje de voz a texto, el cambio de los tamaños de letras etc., permitiendo así equiparación de personas. La accesibilidad en la web para personas con discapacidad está también normalizada a través la Metodología de Evaluación de Conformidad con la Accesibilidad en sitios Web que proporciona una metodología armonizada internacionalmente para la evaluación de sitos web en conformidad con las WCAG $2.0^{7}$.

La accesibilidad virtual, como nueva fase en el proceso de facilitar el acceso a la educación a las personas con discapacidad, propende a cambiar el enfoque de la inclusión para el nivel superior y de pretender ajustar las instituciones a las personas, se debe supeditar el modelo educativo a las personas con discapacidad y ello solo se realiza eficazmente al brindar el servicio educativo directamente en los hogares a través de la educación virtual. Es allí donde ellos tienen las mejores infraestructuras y niveles de accesibilidad las personas. Es allí también por ende donde poder tener acceso al servicio educativo en función de sus intereses. La educación debe llegar a la casa de las personas con discapacidad y no a la inversa que siempre será más lento, limitante y restringido.

6 Esta afirmación proviene de Fanny Wong en el 5to. Congreso Internacional de Educación Superior, Discapacidad y Derechos Humanos - "Visión y Acciones Inclusivas para América Latina" Universidad Tecnológica de Panamá, Panamá. 1-3 de agosto, 2012.

7 Disponible en: <http://examinator.ws/info/metodologia>. 
Sólo así se podrá alcanzar a realizar el derecho a una educación superior individualizada y ajustada a sus especiales capacidades. La educación especial solo se podrá realizar con la educación virtual, y superando el formato tradicional tanto presencial como a distancia, dado que ella no requiere meramente de accesibilidad, sino fundamentalmente de procesos educativos particularizados, individualizados e interactivos con recursos especialmente diseñados de aprendizaje y dinámicas de trabajo colaborativas en redes que les permitan no sentirse en condiciones de minusvalía. En tanto portadores de necesidades especiales, estos sectores requieren una educación individualizada y metodologías, interfaces, recursos, ambientes de aprendizaje, recursos instruccionales y pedagogías especiales atentas a sus particularidades y que no pueden ser realizadas en el marco de la educación de masas ni tampoco en las aulas presenciales sino al interior de los ambientes virtuales. La educación digital en red, en tanto implica una alta flexibilidad, ambientes individualizados de enseñanza focalizados en el autoaprendizaje, se constituye así en el único mecanismo para alcanzar esos objetivos.

Ella se apoya en un conectivismo construccionista y cognitivista, en el aprendizaje en redes colaborativas y en la realización de prácticas a través de aplicaciones informáticas como mecanismos de construcción de competencias. La alta eficacia de metodologías basadas en herramientas informáticas se constituye en la base del autoaprendizaje a través de ambientes virtuales con posible acceso desde los hogares. Varios estudios han mostrado que hoy las personas con discapacidades tienen altos niveles de acceso a la conectividad y a los recursos de las TIC y que en su mayoría no necesita apoyo de este tipo de tecnologías ${ }^{8}$. Pedagogías especiales y tecnologías digitales están altamente entrelazadas. Ellas permiten

\footnotetext{
8 La evaluación de un curso virtual desarrollado en México para personas con necesidades especiales mostró que "en relación con lo tecnológico, las dificultades fueron menores y superadas con rapidez por previa experiencia con el uso de Internet, redes sociales y el mensajero instantáneo". Camacho Real, Claudia y Varela Navarro, Gerardo "Inclusión de personas con discapacidad en la educación virtual: el caso del Bachillerato a Distancia, Universidad de Guadalajara", en Revista Mexicana de Bachillerato a Distancia, Año 5 - № 3, UNAM, México, 2011. Disponible en: <http://www.revistas.unam.mx/index. $\mathrm{php} / \mathrm{rmbd} /$ article/view/29562>.
} 
requerimientos ergonómicos asociadas a los requerimientos particulares, tanto en los casos sincrónicos como asincrónicos, al derivarse de una programación que puede llegar inclusive a ser "a la carte". Las continuas innovaciones en esta materia están facilitando nuevos accesos a medida que se desarrollan y popularizan aplicaciones informáticas específicas para las distintas necesidades especiales, los distintos contenidos de los conocimientos y las diversas habilidades requeridas.

Así, la educación virtual, como lo muestran múltiples experiencias, constituye el medio más idóneo para facilitar los procesos de inclusión educativa a estudiantes que padecen alguna discapacidad, siempre y cuanto la formación pueda adecuarse a través de adaptaciones tecnológicas, académicas y de gestión educativa a los propios particularismo de las discapacidades. Ese camino implica aumentar continuamente el nivel de virtualización de la enseñanza y del aprendizaje sobre la base de que sólo bajo el entorno virtual es que se podrá alcanzar la inclusión y la accesibilidad de todas las diversidades. Los diversos soft y hard educativos se construyen en las intermediaciones reales que permiten el acceso educativo, desde la propia casa de las personas con discapacidad, que finalmente es el único entorno que no restringe la accesibilidad. Pero al tiempo, la soledad y la interacción tienen en la red y en dinámicas colaborativas, las formas más eficientes y también menos discriminantes y marginalizadas. En la red, todos somos y seremos siempre iguales.

\section{Referências}

BRASIL. Censo escolar. Brasília: MEC/INEP, 2006.

CAMACHO REAL, C.; VARELA NAVARRO, G. Inclusión de personas con discapacidad en la educación virtual: el caso del Bachillerato a Distancia. Revista Mexicana de Bachillerato a Distancia, v. 5, n. 3, 2011. Disponible en: <http://www.revistas. unam.mx/index.php/rmbd/article/view/29562>. Accedido el: 20 fev. 2013. 
CEDAC. La integración de las personas con discapacidad en la Educación Superior en Honduras. Tegucigalpa: IESALC, 2004.

CENTRO UNIVERSITÁRIO DE DESARROLLO - CINDA. Deserción y repetición en la educación superior. Santiago: Universidad de Talca; UNESCO/ IESALC, 2005.

JIMÉNEZ SANDOVAL, R. (Ed.). Las personas con discapacidad en la educación superior. San José: Fundación Justicia y Género, 2002.

MATERON PALACIOS, S.; MOLINA BEJAR, R.; PARRA DUSSAN, C. Análisis de la situación de la educación superior para personas con discapacidad en Colombia. Memorias del Primer Foro Virtual Educación Superior Inclusiva. Bogotá: Universidad Autónoma de Manizales, 2010.

MEMbreño, M.; HeRrera, M. E. Propuesta para la Reforma de la Educación especial en el marco de la Reforma educativa Nacional. Honduras: Universidad Nacional Autónoma de Honduras, febrero 2000. mimeo.

ORGANIZACIÓN MUNDIAL DE LA SALUD - OMS - BANCO MUNDIAL. Informe mundial sobre la discapacidad. Malta, 2011. Disponible en: <http://www.who. int/disabilities/world_report/2011/summary_es.pdf>. Accedido el: 5 jul. 2012.

REZENDE, F. A. Educação especial e a EAD. In: LITTO, F.; FORMIGA, M. (Org.). Educação a distância, o estado da arte. São Paulo: ABED - Pearson, 2009. p. 129-140.

RIFKIN, J. El fin del trabajo. Nuevas tecnologías contra puestos de trabajo: El nacimiento de una nueva era. Madrid: Paidos Ibérica, 1996.

VEGA, G. S. et al. Estudio sobre el tratamiento otorgado a personas discapacitadas en las Universidades de Bolivia. Caracas: Universidad NUR IESALC, 2005. 
VENEZUELA. Ministerio de Educación Superior. Apuntes para los términos de referencia de un estudio sobre educación superior y personas con discapacidad. Caraca: Dirección General de Desempeño Estudiantil, 2004.

Recibido: 12/08/2012

Received: 08/12/2012

Aprobado: 25/09/2012

Approved: 09/25/2012 